\title{
Another case of mistaken identity? Vaccinia virus in another live Camelpox vaccine.
}

Zack Saud $^{1}$ and Tariq M. Butt ${ }^{1}$

1- Department of Biosciences, College of Science, Swansea University, Swansea, United Kingdom

\section{Correspondence}

Zack Saud, $\mathrm{PhD}$

Department of Biosciences, College of Science, Swansea University, Singleton Park, Wales, SA2 8PP

Email: zack.saud@gmail.com

\begin{abstract}
Camelpox virus is the causative agent of Camelpox, a highly contagious disease of camels. A high passage Camelpox virus strain has previously been reported to contain several genes which more closely resemble Vaccinia, a virus species with no known natural host, encompassing various strains that show high inter-strain genomic variation. In this study, we demonstrate that yet another high passage, live attenuated vaccine, comprising a different strain of Camelpox virus, contains genomic sequences that match a differing strain of Vaccinia virus. These results are discussed in the context of hypotheses put forward to explain the unknown origins of Vaccinia virus, suggesting further studies to elucidate evolutionary trajectories of Orthopoxviruses through passaging.
\end{abstract}

\section{Keywords}

Orthopox, Vaccinia, Camelpox, Sequencing, Passaging, Attenuation

\section{Introduction}

Camelpox virus (CMLV) is the causative agent of the highly contagious Camelpox disease $[1,2]$, endemic in all regions where camel husbandry is practiced except Australia [1,3]. Several CMLV vaccines are produced worldwide, either in a live attenuated or inactivated adjuvant form [3]. Live attenuated CMLV 
vaccines contain highly passaged live viral particles, produced using methods not much different from those used by Pasteur to develop the first live attenuated vaccine over a century ago [4].

In an earlier study, sequencing of 4 genomic regions of a tissue culture attenuated CMLV isolate (Jouf78), used in a live attenuated vaccine in Saudi Arabia [5], showed that all sequences closely resembled the Vaccinia (VACV) strain Lister [6]. In this study, we show that gene regions from another live attenuated CMLV vaccine, Ducapox, show 100\% identity to the corresponding gene regions of VACV strain Modified Virus Ankara (VACV-MVA).

\section{Methods}

Commercial live attenuated 'Ducapox' vaccines were sourced from Al Bashayer Veterinary Supplies (Dubai, United Arab Emirates), manufactured by Design Biologix (Pretoria, South Africa) and commercialized by Highveld Biological Ltd (Johannesburg, South Africa). The CMLV strain CaPV298-

2, the parent strain of this vaccine, was originally isolated in the United Arab Emirates and attenuated through serial passage in Vero cell culture [7]. Manufacture and expiry dates were 08-2016 and 08-2018 for batch number: DPV0816 (batch 1), and 07-2018 and 06/2019 for batch number DPV0618 (batch 2). Viral titer was stated as $1 \times 10^{6.5} \mathrm{TCID}_{50} / \mathrm{ml}$.

DNA was extracted using the QIAamp DNA Mini kit (Catalog \# 51304, Qiagen, Hilden, Germany), following the DNA purification from tissues protocol, adding $180 \mathrm{uL}$ of Buffer ATL to $25 \mathrm{mg}$ of lyophilized vaccine and following the manufacturer's guidelines with the addition of adding 5 ug of Carrier RNA Poly A (Catalog \# 1017647, Qiagen, Hilden, Germany) to the 200 uL of Buffer AL solution.

Primers designed to amplify the H3L gene region of CMLV comprised; H3L forward primerATGGCGGCGGTGAAAACTC and H3L reverse primer- TTAGATAAATGCGGTAACAAATGTTC. Primers targeting a sub-region of the CMLV DNA polymerase gene (3021 bp full size) were developed using Primer-BLAST [8]: CMLV DNA POL forward primer- AGTCACAAGGGGGTAGGTGG and CMLV DNA POL reverse primer- GCACATATGTTGACGCACCC. This primer set would also amplify 
homologous polymerase gene regions in several Orthopoxvirus species. Another forward primer was designed to better match the gene sequence of VACV-MVA; VACV DNA POL forwardAGTCATAAGGGAGTAGGCGG, differing by 3 nucleotides in comparison to the original CMLV DNA POL forward primer. The reverse primer binding site was identical between both species, hence the original CMLV DNA POL reverse primer was used in conjunction with both forward primers targeting each respective species. The latter primer set showed $100 \%$ complementarity in-silico to several strains of VACV, Cowpox, Horsepox, and Rabbitpox. In order to confirm the absence of CMLV DNA polymerase gene region DNA, individual primer sets specific to the DNA polymerase regions of both CMLV and VACV-MVA were designed using DECIPHER, by inputting the DNA polymerase genes of both species, and keeping the default parameters [9]. The following primer sets were designed for VACV-MVA: VACV DNA POL SP forward- CGGAAAGGATGACGTTGATTTAGCT and VACV DNA POL SP reverse- AATCAGTCGTTCTAACTCTTTCGC. For CMLV, the following primer sets were designed: CMLV DNA POL SP forward- CCATTTAACGCGAGGCCGA and CMLV DNA POL SP reverseTCTCTCTCATAATCCATTTCCGTCAG.

PCR mixtures were prepared in 25 ul volumes using AmpliTaq Gold® 360 Master Mix (Catalog \# 4398876, ThermoScientific, Rochester, USA), following manufacturer guidelines and adding $2 \mathrm{ul}$ of template Ducapox DNA, with initial activation of polymerase at $95^{\circ} \mathrm{C}$ for 10 minutes followed by 40 cycles of DNA denaturation at $95^{\circ} \mathrm{C}$ for 30 seconds, annealing of primers at $50^{\circ} \mathrm{C}$ (and $55^{\circ} \mathrm{C}$ for all reactions targeting the DNA polymerase gene) for 30 seconds and elongation at $72^{\circ} \mathrm{C}$ for 1 minute, with a subsequent elongation hold at $72^{\circ} \mathrm{C}$ for 7 minutes after these cycles.

PCR reaction products were purified using a QIAquick PCR Purification Kit (Catalog \# 28104, Qiagen, Hilden, Germany), following the manufacturer's guidelines. Sanger sequencing of purified amplicons was performed using the BigDye Terminator v3.1 Cycle Sequencing kit (Catalog \# 4337454, ThermoScientific, Rochester, USA) following the manufacturer's guidelines for $10 \mathrm{ul}$ reactions, adding $15 \mathrm{ng}$ of template DNA, with an initial denaturation step at $96^{\circ} \mathrm{C}$ for 1 minute, followed by 25 cycles of 
denaturation at $96^{\circ} \mathrm{C}$ for 10 seconds, annealing at $50^{\circ} \mathrm{C}$ for 5 seconds and extension at $60^{\circ} \mathrm{C}$ for 4 minutes. Purification of reaction mixtures were performed using BigDye XTerminator Purification Kit (Catalog \# 4376484, ThermoScientific, Rochester, USA), following manufacturer's guidelines. Sequencing was done on a SeqStudio Genetic Analyzer (ThermoScientific, Rochester, USA), equipped with a cartridge containing a $28 \mathrm{~cm}$ column (Catalog \# A33671, ThermoScientific, Rochester, USA), using the 'LongSeq_BDX' run module. Sequence analyses was performed using Unipro UGENE software [10] and Genbank accession numbers for all sequences used in this research can be found in Appendix 1.

\section{Results}

The PCR amplification of the vaccine yielded a product of approximately $900 \mathrm{bp}$ for the H3L gene region. For the polymerase gene region, reactions incorporating the CMLV DNA POL primers produced products with lighter bands in comparison to reactions incorporating VACV DNA POL primers, with both products measuring at around 500 bp in size (Fig. 1). Reactions incorporating the CMLV specific primer set CMLV DNA POL SP produced no visible amplicon, whereas reactions incorporating the VACV specific primer set VACV DNA POL SP produced a product band measuring around 900 bp (Fig. 1). There was no observable difference between using a $50^{\circ} \mathrm{C}$ or $55^{\circ} \mathrm{C}$ annealing temperature (Fig. 1).

Length of consensus sequences produced for each sample and percentage identity of consensus sequences to the corresponding genomic position of VACV-MVA are shown in table 1. Consensus sequences for each sample can be found in Appendix 2. Genomic mapping graphs for each sample, including the H3L gene region mapped to CMLV, can be found in Appendix 3. All sequenced amplicons showed 100\% identity to corresponding gene regions in VACV-MVA.

\section{Discussion}

We confirmed the absence, to the sensitivity of the PCR method, of CMLV polymerase gene region DNA in the Ducapox vaccine, a gene involved in viral replication and known to be relatively conserved within 
the Orthopoxvirus genus [11]. Our findings corroborate the findings of Yousif et al. 2012 [6], who showed that four gene regions of a tissue culture attenuated CMLV (isolate Jouf-78), more closely resembled VACV, albeit strain Lister, whereas the Ducapox strain in this study more closely resembles VACV-MVA. Given the nucleotide differences between the sequenced gene regions of these VACV strains, it is unlikely that both seed viruses came from the same misidentified stock, or that the observations are the result of a circulating VACV strain which was isolated, misidentified, and used as seed stocks for both vaccines.

Yousif et al. 2012 [6] attribute a possible contamination event to their findings. Although misidentification of initial seed stock or contamination would also be a plausible explanation for our findings, given that this is the second strain of CMLV found to resemble VACV after passage, other possibilities should be considered for the following reasons. Firstly, the Ducapox CMLV strain was claimed to have been cultured in Vero cells, a continuous cell lineage, originally isolated from kidney epithelial cells extracted from an African green monkey [7]. VACV-MVA cannot be cultured in most mammalian cells, or produces minimal viral titers in the few known permissive mammalian cell types in which it can be cultured [12]. Secondly, the question of whether an Othropox virus can be passaged to create a more VACV-like strain is not a novel one, VACV has no known natural host, and the definitive origins of the virus are still highly contested, with many hypotheses having been put forward [13]. Better conclusions could be made from conducting whole genome sequencing of high passage strains and doing whole genome comparisons. Also, it would be interesting to see the evolutionary trajectory with passage, at the whole-genome level, of the same seed stock of Orthopoxvirus grown in different mammalian and/or avian cell lines. Whole genome sequence analyses of CMLV passages at differing passage intervals would determine the evolution of the virus through the passage stages. Deep-sequencing would also show the abundance of viral quasi-species within the sample. However unlikely it may be, it cannot be discounted that divergent Orthopoxviruses may gradually evolve to become more VACV like with greater passage, especially given the fact that genomes of most VACV strains tend to be smaller than those of 
pathogenic Orthopoxviruses, with high inter-strain genomic variation [14]. This theory could be tested experimentally, as one could examine whether high passaging of a number of the most diverged Orthopoxvirus species causes them to separately evolve to become more VACV-like. This may certainly hold true for CMLV, as some strains of VACV are thought to have been derived from attenuating VARV, and, amongst the Orthopoxviruses, CMLV shows the closest genetic homology to VARV [15].

To conclude, this paper has shown that another highly passaged live attenuated CMLV vaccine contains genomic regions that match $\mathrm{VACV}$, highlighting the need for more robust and stringent testing of biologicals intended for veterinary applications.

\section{Conflicts of Interest}

None

\section{Acknowledgement}

This research did not receive any specific grant from funding agencies in the public, commercial, or notfor-profit sectors. We thank staff at the University of Sharjah for facilitating the use of their facilities. 
Table 1: Lengths of consensus sequences produced for each primer set and percentage identity of the consensus sequences to the corresponding genomic position of VACV-MVA. No sequencing was attempted with primer sets CMLV DNA POL SP due to the lack of visible amplicon formation following PCR.

\begin{tabular}{lcccc}
\hline Primer set & Batch & $\begin{array}{l}\text { Length of consensus } \\
\text { sequence }\end{array}$ & $\begin{array}{l}\text { Genomic position of } \\
\text { VACV-MVA }\end{array}$ & $\begin{array}{l}\text { \% identity to } \\
\text { VACV-MVA }\end{array}$ \\
\hline H3L & 1 & 879 & $83211-84080$ & 100 \\
& 2 & 870 & $83211-84080$ & 100 \\
CMLV DNA Pol & 1 & 507 & $50102-50608$ & 100 \\
& 2 & 464 & $50102-50565$ & 100 \\
VACV DNA Pol & 1 & 507 & $50102-50608$ & 100 \\
& 2 & 507 & $50102-50608$ & 100 \\
VACV DNA Pol SP & 1 & 829 & $49308-50136$ & 100 \\
& 2 & 818 & $49319-50136$ & 100 \\
CMLV DNA Pol SP & \multicolumn{4}{c}{ No amplicon produced } \\
\hline
\end{tabular}

Figure 1: DNA polymerase gene region amplicons of Ducapox vaccine DNA. Reactions incorporating the CMLV DNA POL primers produced products with lighter bands in comparison to reactions incorporating VACV DNA POL primers, with both products measuring at around $500 \mathrm{bp}$ in size. Reactions incorporating the CMLV specific primer set CMLV DNA POL SP produced no visible amplicon. Reactions incorporating the VACV specific primer set VACV DNA POL SP produced a product band measuring around $900 \mathrm{bp}$. There was no observable difference between using a $50^{\circ} \mathrm{C}$ or $55^{\circ} \mathrm{C}$ annealing temperature.

\section{References}

1. Tadesse T, Mulatu E, Bekuma A. Review on Camel Pox: An Economically Overwhelming Disease of pastorals. Int. J. Adv. Res. Biol. Sci. 2018; 5(9):65-73.

2. Zhu S, Zimmerman D, Deem SL. A Review of Zoonotic Pathogens of Dromedary Camels. EcoHealth 2019; 1-22.

3. OIE Manual of Diagnostic Tests and Vaccines for Terrestrial Animals. 2009; Camelpox. Chapter 2.9.2.: 1177-1184.

4. Pasteur L, Chamberland C, Roux E. De l'attenuation des virus et de leur retour a la virulence. C. R. Acad. Sci, 1881; 92:430-435.

5. Hafez SM, Al-Sukayran A, Dela Cruz D, Mazloum KS, Al-Bokmy AM, Al-Mukayel A, Amjad AM. Development of a live cell culture camelpox vaccine. Vaccine 1992; 10(8):533-9.

6. Yousif AA, Al-Ali AM. A case of mistaken identity? Vaccinia virus in a live camelpox vaccine. Biologicals 2012; 40(6):495-8. 
7. Kaaden DR, Walz CP, Czerny U, Wernery U, Allen WR. Progress in the development of a camel pox vaccine. 1992 Proceeding of the 1st Int. Camel Conference, 47-49.

8. Ye J, Coulouris G, Zaretskaya I, Cutcutache I, Rozen S, Madden TL. Primer-BLAST: A tool to design target-specific primers for polymerase chain reaction. BMC Bioinformatics. 2012; 13:134. doi:10.1186/1471-2105-13-134.

9. ES Wright, Yilmaz LS, Ram S, Gasser JM, Harrington GW, Noguera DR. Exploiting Extension Bias in PCR to Improve Primer Specificity in Ensembles of Nearly Identical DNA Templates. Environ Microbiol. 2014; 16(5):1354-65 doi:10.1111/1462-2920.12259.

10. Okonechnikov K, Golosova O, Fursov M, UGENE team. Unipro UGENE: a unified bioinformatics toolkit. Bioinformatics 2012; 28:1166-1167.

11. Moss B. Poxvirus DNA replication. Cold Spring Harb Perspect Biol. 2013; 5:a010199.

12. Meyer H, Sutter G, Mayr A. Mapping of deletions in the genome of the highly attenuated vaccinia virus MVA and their influence on virulence. J. Gen. Virol. 1991; 72:1031-1038.

13. Baxby D. The origins of Vaccinia virus. The Journal of Infectious Diseases. 1977; 136(3):453455.

14. Qin L, Favis N, Famulski J, Evans DH. Evolution of and evolutionary relationships between extant vaccinia virus strains. J Virol. 2015; 89(3):1809-24.

15. Afonso CL, Tulman ER, Lu Z, Zsak L, Sandybaev NT, Kerembekova UZ, Zaitsev VL, Kutish, GF, Rock DL. The genome of camelpox virus. Virology 2002; 295(1):1-9. 


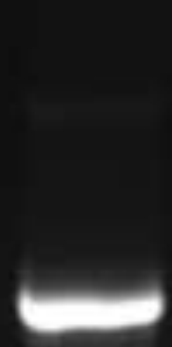

$500 \mathrm{bp}$ 